
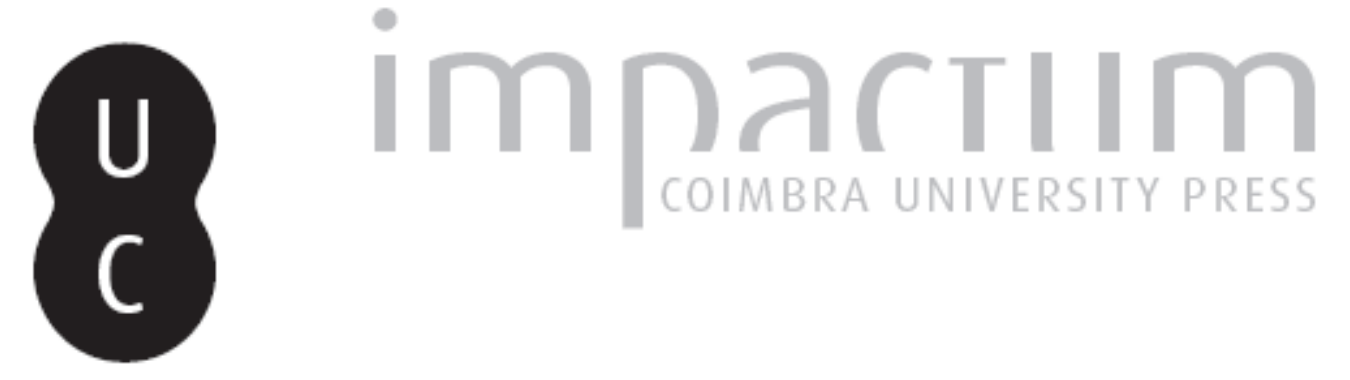

\title{
O impacto do ambiente nos níveis de obesidade infantil: quando o território emerge como um fator de diferenciação e risco
}

\author{
Autor(es): $\quad$ Nogueira, H.; Ferrão, M. M.; Gama, A.; Grespan, F.; Mourão, I.; \\ Marques, V. Rosado; Padez, C.
} Publicado por: $\begin{aligned} & \text { Faculdade de Letras da Universidade de Coimbra, Departamento de } \\ & \text { Geografia }\end{aligned}$

URL persistente:

DOI: $\quad$ DOI:http://dx.doi.org/10.14195/0871-1623_31_21

Accessed : $\quad$ 26-Apr-2023 14:08:10

A navegação consulta e descarregamento dos títulos inseridos nas Bibliotecas Digitais UC Digitalis, UC Pombalina e UC Impactum, pressupõem a aceitação plena e sem reservas dos Termos e Condições de Uso destas Bibliotecas Digitais, disponíveis em https://digitalis.uc.pt/pt-pt/termos.

Conforme exposto nos referidos Termos e Condições de Uso, o descarregamento de títulos de acesso restrito requer uma licença válida de autorização devendo o utilizador aceder ao(s) documento(s) a partir de um endereço de IP da instituição detentora da supramencionada licença.

Ao utilizador é apenas permitido o descarregamento para uso pessoal, pelo que o emprego do(s) título(s) descarregado(s) para outro fim, designadamente comercial, carece de autorização do respetivo autor ou editor da obra.

Na medida em que todas as obras da UC Digitalis se encontram protegidas pelo Código do Direito de Autor e Direitos Conexos e demais legislação aplicável, toda a cópia, parcial ou total, deste documento, nos casos em que é legalmente admitida, deverá conter ou fazer-se acompanhar por este aviso.

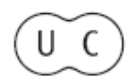




\title{
O impacto do ambiente nos níveis de obesidade infantil: quando o território emerge como um fator de diferenciação e risco
}

\author{
H. Nogueira \\ Centro de Investigação em Antropologia e Saúde e Departamento de Geografia. Faculdade de Letras da Universidade de Coimbra. \\ helenamarquesnogueira@hotmail.com
}

\section{M. Ferrão}

Departamento de Ciências da Vida e Centro de Investigação em Antropologia e Saúde. Universidade de Coimbra. Departamento de Ciências da Vida, Universidade de Coimbra.

\author{
A. Gama \\ Faculdade de Ciências da Universidade de Lisboa. Centro de Investigação em Antropologia e Saúde. Universidade de Coimbra.

\section{F. Grespan} \\ Centro de Investigação em Antropologia e Saúde. Universidade de Coimbra.
}

\section{Mourão}

Universidade de Trás-os-Montes e Alto Douro.

\section{Rosado Marques}

Instituto de Investigação Científica Tropical e Centro de Investigação em Antropologia e Saúde. Universidade de Coimbra.

\section{Padez}

Departamento de Ciências da Vida e Centro de Investigação em Antropologia e Saúde. Universidade de Coimbra.

\section{Resumo:}

Este estudo investiga as associações entre as percepções parentais do ambiente construído e social do local de residência e a prevalência de obesidade infantil segundo o género das crianças. Estuda-se ainda a oferta de modalidades desportivas infantis, verificando a sua potencial contribuição para os valores observados de excesso de peso/ obesidade. Numa primeira fase, observaram-se 1885 crianças em idade escolar (3-10 anos) residentes no distrito de Coimbra. Nesta amostra, verificou-se uma associação significativa entre a percepção parental do ambiente construído e social e os valores de obesidade no sexo feminino. Numa segunda fase, verificou-se que a oferta de modalidades desportivas na cidade de Coimbra é desequilibrada, restringindo fortemente as oportunidades de prática desportiva às crianças do sexo feminino. Em 67 oportunidades de prática desportiva observadas na cidade, verificou-se que 32 eram mistas, 23 eram direccionadas para o sexo masculino e 12 para o sexo feminino. Conclui-se que as crianças do sexo feminino sofrem processos de discriminação ambiental cujo impacte na saúde pode ser nefasto. Neste contexto, argumenta-se a necessidade de intervenções ambientais, não só como forma de promoção da saúde, mas também de equidade entre os géneros.

Palavras-chave: Obesidade infantil. Ambiente. Modalidades desportivas. Equidade.

\section{Résumé:}

L'impact de l'environnement sur les niveaux d'obésité infantile : quand le territoire émerge comme un facteur de différenciation et de risque.

Cet étude recherche les associations entre les perceptions parentales de l'environnement construit et social de l'endroit de résidence et la prévalence de l'obésité infantile selon le genre des enfants. On étude aussi la disponibilité de modalités sportives infantiles, en vérifiant leur possible contribution pour les valeurs observées d'excès de poids/ obésité. Dans une première phase os a observé 1885 enfants en âge préscolaire (3-10 ans), résidants au district de Coimbra. Dans cet échantillon on a verifié une association significative entre la perception parentale de l'environnement construit et social et les valeurs d'obésité au sexe féminin. Dans une deuxième phase os a verifié que la disponibilité de 
modalités sportives à ville de Coimbra est déséquilibrée, en restreignant fortement les opportunités de pratique sportive aux enfants du sexe féminin. Dans 67 opportunités de pratique sportive observées à cette ville-là, on a vérifié que 32 étaient mixtes, 23 étaient dirigées pour le sexe masculin et 12 pour le sexe féminin. On conclut que les enfants du sexe féminin souffrent des procédés de discrimination environnementale, dont l'impact sur la santé peut être néfaste. Dans ce contexte-ci, on argument le besoin d'interventions environnementales, comme uns manière de promouvoir la santé et aussi l'équité entre les genres.

Mots-clés: Obésité infantile. Environnement. Modalités sportives. Équité.

\section{Abstract:}

The impact of the environment on levels of childhood obesity: when the territory emerges as a differentiating and risk factor

This study investigates the links between parents' perceptions of social and built local environment and child sex-specific levels of excess weigh/obesity. Further, we analyse if the availability of child sports is a potential contribute to the observed patterns of excess weight/obesity. In a first step, we observed 1885 school children (aged 3-10 yrs) living in Coimbra district. In this sample, results have shown a significant association between parental perceptions of built and social local environment and obesity in girls. In a second step, we found that the availability of sports in Coimbra city is unbalanced, heavily restricting the opportunities of sports practice to girls. From the 67 sports opportunities observed in Coimbra, 32 were directed to both sexes, 23 were directed towards boys and 12 to girls. We've concluded that girls experience processes of environmental discrimination, which may have a harmful health impact. In this context, we argue the need for environmental interventions, not only as a means of promoting health, but also gender equity.

Keywords: Childhood obesity. Environment. Sports. Equity.

\section{Introdução}

As prevalências da obesidade têm vindo a aumentar, de forma gradual e global, desde a década de 80 (FInUCANE et al., 2011), atingindo atualmente níveis epidémicos. Segundo a OMS, esta prevalência triplicou nos últimos 25 anos, tornando-se a obesidade uma das maiores causas de morte evitável no mundo desenvolvido (WHO, 2007). Esta tendência de aumento de peso atinge de forma quase transversal todos os grupos populacionais, verificando-se tanto em crianças, como em adultos. Para os adultos europeus, estima-se que o excesso de peso seja responsável por $50 \%$ dos casos de hipertensão e 33\% dos casos de enfarte do miocárdio (Mladovsky et al., 2009). Já as consequências da obesidade infantil são mais insidiosas e, muitas vezes, apenas quantificáveis a longo prazo. Todavia, vários estudos têm apontado um vasto leque de efeitos nefastos na saúde, quer precoces, quer tardios, como a diabetes de tipo II, a hipertensão e a hipercolesterolémia (WHO, 2000; Wang e Lobstein, 2006; Wang e Dietz 2002). Para a obesidade infantil, Portugal apresenta umas das situações mais graves de toda a Europa. Num estudo desenvolvido em crianças dos 7 aos 9 anos, PADEz et al. (2004) concluem que a prevalência de obesidade infantil em Portugal é de $31,5 \%$, valor superior ao apresentado pela
Grécia (31\%) e Espanha (30\%), e apenas suplantado pelo de Itália (36\%).

Será esta tendência de aumento de peso inevitável, revelando-se quase como um sinal do desenvolvimento? Como pode a humanidade invertê-la?

A pluricausalidade da obesidade é hoje consensual: admite-se a influência da componente genética, mas sabe-se que esta não pode explicar o recente aumento no número de casos (СoHen et al., 2006).

\section{Ambiente obesogénico}

Estudos realizados nesta temática sugerem que alguns factores do ambiente construído e social estão subjacentes ao aumento de peso referido, uma vez que promovem o aporte calórico e/ou desencorajam o gasto de energia na atividade física quotidiana (GILES-CoRTI e Donovan, 2003; Portinga 2006). Os comportamentos alimentares e de atividade física das crianças são influenciados por uma variedade de factores ambientais, podendo destacar-se as infra-estruturas desportivas e de lazer existentes nos seus espaços de vida, a oferta de determinados alimentos (disponibilidade de fast food ou de alimentos saudáveis), o acesso ao transporte público e ao transporte ativo, as condições para caminhar e andar a pé (desde a existência de passeios até à sinalização e iluminação), a segurança real e percep- 
cionada, tanto a rodoviária (neste caso relacionada com a intensidade e velocidade do tráfego automóvel e possível de ser aumentada quer com restrições - por exemplo, rotundas e sinalização - quer com facilidades direccionadas ao pedestre - passadeiras, viadutos, etc), como a criminal, dependendo esta última, muitas vezes, do ambiente social, nomeadamente da coesão social e do capital social (Frank et al., 2004; CoHen et al., 2006; KIm et al., 2006; CERIN et al., 2007; Frank et al., 2007; LESLIE et al., 2007). Estes factores ambientais, em interacção, estruturam os designados "ambientes obesogénicos", uma vez que condicionam o equilíbrio entre gastos e aportes energéticos.

\section{Obesidade, ambiente e desigualdades}

À semelhança do que acontece com muitas outras patologias, a obesidade é discriminativa de clivagens e desigualdades existentes na sociedade. Regra geral, indivíduos de mais baixo estatuto socioeconómico, mais vulneráveis e com pior estado de saúde, têm maior probabilidade de residir em áreas carenciadas, onde falham os recursos e as facilidades que poderiam promover a sua saúde, mas onde abundam aspectos que a podem fragilizar (MACINTYRE, 2007; Nogueira, 2010). Esta ideia da sobreposição de riscos individuais e ambientais, que jogam em interacção e multiplicação, tem sido estudada sob diferentes nomes: injustiça ambiental ("environmental injustice") (Buzzeluı, 2007; Maroko et al., 2009), modelos de amplificação da privação ("deprivation amplification models") (MACINTYRE, 2007; NogueIra, 2010).

Todavia, para além da diferenciação socioeconómica, que será oportunamente tratada, verifica-se uma desigualdade de género na prevalência de obesidade infantil que, regra geral, penaliza o sexo feminino (PADEZ et al., 2004). Alguns autores, procurando compreender os maiores valores de obesidade verificados nas meninas, sublinharam a existência de um padrão de influências ambientais específicas segundo o género. A este propósito, Hume et al. (2005), Evenson et al. (2006) e SANTOS et al. $(2008,2009)$ referem-se ao maior impacte dos ambientes obesogénicos para o sexo feminino, verificado em adolescentes e em mulheres adultas. Porém, nenhum estudo procurou averiguar a existência de uma influência ambiental específica ainda na infância.

O objectivo do presente estudo é, numa primeira fase, analisar a existência de associações entre a percepção parental do ambiente físico e social das áreas de residência das crianças com idades compreendidas entre os 3 e os 10 anos, residentes no distrito de Coimbra, e os seus níveis de obesidade, averiguando a exis- tência de uma especificidade de género. Numa segunda fase procede-se à observação, para a cidade de Coimbra, da oferta de modalidades desportivas, nomeadamente da diversidade e quantidade disponíveis para o grupo etário em estudo, verificando se essa oferta pode contribuir para os padrões observados na prevalência da obesidade por género. A hipótese subjacente é a de que o ambiente pode estar a assumir um papel perverso, ao discriminar as crianças segundo o seu género, restringindo-thes a oportunidade de praticar atividades desportivas e de desenvolver estilos de vida saudáveis. É, neste sentido, um estudo inovador, sendo o primeiro que se preocupa em explorar de forma sistemática as diferenças de género nas questões da obesidade infantil versus ambiente.

\section{Material e métodos}

\section{Amostra}

Os dados utilizados neste estudo foram recolhidos entre Março de 2009 e Julho de 2010, em 23 escolas do ensino pré-escolar e do $1^{\circ}$ ciclo do Ensino Básico público e privado do distrito de Coimbra. Foram observadas 1885 crianças - 933 meninos e 952 meninas - entre os 3 e os 10 anos de idade.

\section{Medidas antropométricas}

Em cada escola, dois técnicos devidamente preparados avaliaram as crianças utilizando procedimentos standards. A altura foi medida com as crianças descalças, utilizando um estadiómetro e o peso com as crianças vestindo o mínimo de roupa possível, utilizando uma balança electrónica com precisão de $100 \mathrm{~g}$. O Índice de Massa Corporal (IMC) foi calculado como peso/estatura ${ }^{2}$ $\left(\mathrm{Kg} / \mathrm{m}^{2}\right)$. As definições de excesso de peso e de obesidade foram baseadas nos intervalos definidos pelo International Obesity Task Force (Cole et al., 2000), tendo as crianças sido agrupadas em duas categorias: peso normal versus excesso de peso/obesidade

\section{Estatuto socioeconómico}

Os pais das crianças foram convidados a preencher um questionário sobre diversas características familiares, do alojamento e da área de residência. Com base neste questionário, avaliou-se o estatuto socioeconómico (ESE), recorrendo aos anos de educação parental. Utilizaram-se ambos os níveis de educação, do pai e da mãe, atendendo a dois factores: por um lado, a literatura científica, que aponta a educação da mãe como o mais importante indicador de ESE (RUNDLE et al., 
2009); por outro lado, a sociedade portuguesa, na qual o homem é ainda, tradicionalmente, o chefe de família, determinando com frequência as condições de vida do agregado familiar. Esta variável de ensino formal foi categorizada em 5 grupos: até 4 anos, entre 4 e 6 anos, entre 7 e 9 anos, secundário/profissional $\left(10^{\circ}, 11^{\circ}\right.$ e $12^{\circ}$ anos) e universitário.

\section{Ambiente (percepção parental da área de residência)}

Para avaliar as características ambientais podem ser utilizados diferentes tipos de dados: dados objectivos, ou directos, resultantes de auditorias e da aplicação dos SIG's e dados subjectivos, que avaliam a forma como os indivíduos percepcionam o seu ambiente. Vários estudos, avaliando a pertinência e precisão destas diferentes medidas ambientais, concluíram pela maior eficácia das medidas subjectivas quando utilizadas em estudos de associação entre ambiente e comportamentos sedentários (IRVING et al., 2005; McGINN et al., 2007). Defende-se que estudos focados em crianças devem considerar e até privilegiar as percepções parentais, uma vez que as decisões dos pais afectam toda a família, desde o local em que residem até aos espaços frequentados pelas crianças e às atividades por estas desenvolvidas (SAllis et al., 2000; CARVER et al., 2010).

As percepções parentais foram avaliadas através do questionário já referido, utilizando um módulo adaptado do International Physical Activity Prevalence Study (IPS, 2002). Este questionário tem sido amplamente utilizado, comprovando-se a sua consistência e adequabilidade do instrumento, inclusive para a realidade portuguesa (MOTA et al., 2005; SANTOs et al., 2009). O questionário é composto por 17 questões, das quais se seleccionaram as que apresentavam uma escala de Likert, composta por 4 níveis: discordo completamente; discordo de certa forma; concordo de certa forma; concordo completamente. As 15 questões seleccionadas direccionam-se a distintos aspectos do ambiente físico e social, nomeadamente: disponibilidade e proximidade de diversas infra-estruturas, equipamentos e serviços locais; disponibilidade e manutenção de infra-estruturas de suporte ao transporte ativo; qualidades estéticas; conectividade da rede viária; acesso ao transporte público; segurança (rodoviária e criminal); ambiente social (coesão social, relações de vizinhança).

\section{Ambiente (disponibilidade de modalidades desportivas)}

Como referido anteriormente, procurou averiguarse a hipótese da existência de um processo de discrimi- nação ambiental específica segundo o género, tomando como estudo de caso a cidade de Coimbra. Para tal, procedeu-se ao levantamento de todos os pontos na cidade com oferta de desporto formal direccionado a crianças até aos 10 anos. Em cada um desses pontos - associações desportivas, recreativas e socioculturais, ginásios, clubes, academias, etc. - recolheu-se, por inquérito, as modalidades oferecidas às crianças e o número de crianças que as frequentavam em 2009/2010, por sexo.

\section{Procedimentos estatísticos}

A análise dos dados iniciou-se com a verificação da existência de diferenças nos níveis de excesso de peso/obesidade das crianças em função da sua idade e do estatuto socioeconómico dos pais, efetuada por intermédio de testes do qui-quadrado.

As variáveis ambientais foram correlacionadas, entre si e com as categorias de IMC estabelecidas, através de coeficientes de correlação de Spearman, verificando-se a existência de várias correlações fortes e significativas, que sugeriram a possibilidade de reduzir os dados. A redução da informação ambiental foi efetuada pela aplicação de uma Análise em Componentes Principais para Dados Categóricos (CATPCA). 0 resultado principal da CATPCA é um número reduzido de dimensões, não correlacionadas, que encerram, em si, a maior parte da informação contida nas variáveis originais. As dimensões foram seleccionadas, tendo sido rejeitadas as consideradas irrelevantes pela aplicação do critério de Kaiser (valor próprio inferior a 1). Cinco dimensões cumpriam este critério inicial, mas apenas duas revelaram possuir adequada consistência interna, avaliada pelos coeficientes Alpha de Cronbach (superiores a 0,5) (SAntos, 1999; SAntos et al., 2008). Estas dimensões foram melhoradas, maximizando-se a sua consistência e confiabilidade, pela eliminação das variáveis que, em cada dimensão, apresentassem saturações mais baixas (Cummins et al., 2005; NogueirA, 2009). A melhor solução reteve duas dimensões, compostas por 13 variáveis e explicando cerca de $55 \%$ do total da variância:

1. Dimensão do ambiente construído (uso do solo e desenho urbano): avalia a disponibilidade e proximidade de infra-estruturas e recursos (comércio, equipamentos de desporto e lazer), a acessibilidade a destinos úteis e interessantes (equipamentos vários, serviços), a disponibilidade e manutenção de características físicas de suporte ao transporte ativo, como passadeiras, passeios, ciclovias e a estética geral dos lugares (coeficiente $\alpha$ de Cronbach $=0,8$ ). 
2. Dimensão do ambiente social e da segurança: engloba variáveis relacionadas com a percepção de desordem social, falta de controlo social, crime, violência e segurança, incluindo a segurança rodoviária (coeficiente $\alpha$ de Cronbach $=0,6$ ).

Para efeitos de análise estatística, as dimensões foram dicotomizadas com base na sua mediana, tendo sido categorizadas em percepção geral negativa versus percepção geral positiva.

A associação entre as categorias de IMC das crianças e as percepções parentais da área de residência foi analisada por intermédio de regressões logísticas binárias. Desenvolveram-se dois modelos, um para cada género, que foram progressivamente ajustados pela idade e ESE dos pais (possíveis variáveis confundentes). Para cada género, obtiveram-se as Odds Ratios, ajustadas pela idade e ESE, e ainda os respectivos intervalos de confiança a 95\%. Calcularam-se também os erros padrão robustos, que permitem considerar o efeito do agrupamento das crianças em cada escola (clustering da escola, também este potencialmente gerador de "ruído" nos modelos desenvolvidos).

A informação sobre o tipo e número de modalidades desportivas foi utilizada para se verificar a discrepância entre a oferta de modalidades adequadas aos diferentes géneros (diversidade e disponibilidade de locais de prática). Apesar de não se possuir ainda informação completa relativa às crianças inscritas em todas as modalidades, esta está disponível para a maioria das atividades desportivas existentes, o que permitiu diferenciar as atividades em mistas (frequência possível por ambos os géneros), tendencialmente masculinas (frequência preferencial - possível ou observada - por crianças do sexo masculino) e tendencialmente femininas (frequência preferencial - possível ou observada - por crianças do género feminino).

Toda a análise estatística foi efetuada com o programa SPSS 17.0.

\section{Resultados}

A prevalência de excesso de peso e obesidade registada na amostra foi de $26,8 \%$ (21,5\% para excesso de peso e $5,3 \%$ para obesidade), sendo os valores observados nas meninas mais elevados do que os registados nos meninos (respectivamente $12,5 \%$ e $2,8 \%$ para excesso de peso e obesidade nas meninas; e $9,0 \%$ e $2,5 \%$ para os meninos) (Quadro I).

Analisando a associação entre excesso de peso/ obesidade das crianças em função do nível de instrução dos pais, verifica-se a existência de um claro gradiente social: obesidade e excesso de peso vão tendencialmente aumentando com a diminuição do nível de instrução (Quadro I). As diferenças encontradas nas prevalências de excesso de peso e obesidade por nível de instrução são significativas, independentemente de se considerar a instrução do pai ou da mãe. A diferença entre as categorias extremas é maior considerando a instrução do pai; o rácio entre estas categorias é de 1,46, considerando a instrução da mãe, atingindo 1,74, para a instrução do pai.

O Quadro II apresenta os resultados dos modelos de regressão logística binária que avaliam a associação entre a percepção parental do ambiente e a obesidade nos dois sexos.

Verifica-se que, após ajustamento para algumas das variáveis que podem interferir na associação referida (variáveis ditas confundentes, como a idade, a instrução parental e o cluster de escola), as crianças do sexo feminino cujos pais reportam uma percepção negativa do ambiente construído (equipamentos e recursos, acesso a destinos úteis e interessantes, disponibilidade e manutenção de características físicas de suporte ao transporte ativo e estética) possuem maior probabilidade de apresentar excesso de peso e obesidade $(47 \%$, OR $=1,47 ; p<0.05)$.

Para este género, a influência da percepção parental do ambiente social revelou-se igualmente significativa: meninas que residem em áreas consideradas inseguras e potencialmente perigosas pelos seus pais têm uma probabilidade $34 \%$ maior de apresentarem

Quadro I

Características da amostra

\begin{tabular}{|c|c|c|}
\hline & $\begin{array}{c}\text { Peso normal } \\
\text { (\%) }\end{array}$ & $\begin{array}{c}\text { Excesso de } \\
\text { peso/obesidade } \\
(\%)\end{array}$ \\
\hline Total & 73.3 & 26.8 \\
\hline Sexo masculino $(n=933)$ & 88.5 & 11,5 \\
\hline Sexo feminino $(n=952)$ & 84.7 & 15,3 \\
\hline \multicolumn{3}{|l|}{ Instrução do pai } \\
\hline $1-4$ anos & 65.2 & 34.8 \\
\hline 5-6 anos & 71.2 & 28.8 \\
\hline 7-9 anos & 69.3 & 30.7 \\
\hline $10-12$ anos & 73.0 & 17.0 \\
\hline$>12$ anos & 80.0 & 20.0 \\
\hline \multicolumn{3}{|c|}{$X^{2}=18,4 ; p=0.001$} \\
\hline \multicolumn{3}{|l|}{ Instrução da mãe } \\
\hline 1-4 anos & 66.2 & 33.9 \\
\hline 5-6 anos & 67.4 & 32.6 \\
\hline 7-9 anos & 69.5 & 30.5 \\
\hline $10-12$ anos & 75.0 & 25.0 \\
\hline$>12$ anos & 76.8 & 23.2 \\
\hline
\end{tabular}


Quadro II

Odds ratios (OR) e Intervalos de Confiança (IC) a 95\% resultantes dos modelos de regressão logística binária estimando a probabilidade das crianças revelarem excesso de peso/obesidade

\begin{tabular}{|c|c|c|c|c|}
\hline & \multicolumn{2}{|c|}{ Meninos } & \multicolumn{2}{|c|}{ Meninas } \\
\hline & OR $_{\text {ajustadas }}{ }^{\mathrm{b}}$ & IC $95 \%$ & OR ajustadas ${ }^{b}$ & IC95\% \\
\hline \multicolumn{5}{|c|}{ Ambiente construído (uso do solo e desenho urbano) } \\
\hline Percepção geral positiva ${ }^{a}$ & 1 & & 1 & \\
\hline Percepção geral negativa & 0.839 & $0.543-1.318$ (n.s.) & 1,47 & $1.026-2.105^{*}$ \\
\hline \multicolumn{5}{|l|}{ Ambiente social e segurança } \\
\hline Percepção geral positiva ${ }^{a}$ & 1 & & 1 & \\
\hline Percepção geral negativa & 1.004 & $0.812-1.243$ (n.s.) & 1.339 & $1.035-1.732^{*}$ \\
\hline
\end{tabular}

a Categoria de referência

${ }^{\text {b} A j u s t a d a s ~ p a r a ~ i d a d e, ~ i n s t r u c ̧ a ̃ o ~ d o ~ p a i ~ e ~ d a ~ m a ̃ e ~ e ~ c l u s t e r ~ d e ~ e s c o l a ~}$

${ }^{*} p<0.05$

peso excessivo e obesidade $(\mathrm{OR}=1,339 ; \mathrm{p}<0.05)$, por comparação com as meninas que residem em áreas consideradas "convenientes", adequadas, socialmente organizadas e seguras.

Estas associações não apresentaram significância estatística para as crianças do sexo masculino.

O Quadro III apresenta o resumo da oferta de modalidades de desporto formal (tipo de atividade disponível e quantidade da oferta) para crianças até aos 10 anos existente na cidade de Coimbra.

Uma análise ao quadro evidencia a grande diferença existente na oferta de modalidades desportivas adequadas ou preferidas pelos diferentes géneros, atendendo tanto à sua diversidade, como à sua quantidade. Assim, quanto à diversidade de modalidades desportivas, registaram-se 10 modalidades de frequência mista, 8 de frequência tendencialmente masculina ${ }^{1}$ e somente 3 de frequência tendencialmente feminina. Também o número de modalidades - ou seja, a sua disponibilidade nas coletividades e instituições inquiridas, condicionante da oportunidade de prática - varia fortemente segundo o género, registando-se 32 oportunidades de prática para as modalidades de tipo misto, 23 oportunidades de prática para as modalidades tendencialmente masculinas, mas somente 12 oportunidades de prática para as modalidades tendencialmente femininas.

\section{Discussão}

Os resultados mostraram que excesso de peso e obesidade afetam mais as crianças do sexo feminino, comparativamente às do sexo masculino. Revelaram também que possuir peso excessivo ou ser obeso é uma

${ }^{1}$ Refira-se que algumas das atividades classificadas como tendencialmente masculinas podem ser frequentadas por meninas, não se verificando, contudo, nenhuma inscrição feminina (ver quadro III), enquanto noutras essa frequência não é possível, uma vez que não existem equipas femininas (casos do hóquei e do rugby). condição que está, de certa forma, embutida nas estruturas sociais, uma vez que apresenta um gradiente social forte e significativo. Estes resultados corroboram estudos anteriores, inclusive realizados em Portugal (PADEZ et al., 2004).

Os resultados revelaram também associações entre as percepções parentais do ambiente construído e social e as categorias de IMC nas crianças do sexo feminino, associações estas que não foram observadas para as crianças do sexo masculino. Verificou-se ainda que a disponibilidade de modalidades desportivas existentes na cidade de Coimbra penaliza fortemente as crianças do sexo feminino, atendendo tanto à sua diversidade, como à sua quantidade. Estes resultados não podem ser confrontados com outros resultados já obtidos, uma vez que não se conhecem estudos semelhantes anteriormente publicados.

Relativamente às associações entre percepções parentais e peso das crianças, TIMPERIo et al. (2004), Burdette et al. (2003) e CARVER et al. (2010), que estudaram amostras de crianças com idades comparáveis às do presente estudo, não se debruçaram sobre as diferenças de género. Somente alguns estudos realizados em adolescentes sugeriram uma influência ambiental específica segundo o género. Hume et al. (2005), Evenson et al. (2006) e SANTOS et al. (2009) concluem que as raparigas adolescentes parecem mais sensíveis a algumas características do ambiente construído, enquanto os rapazes mostram uma particular sensibilidade ao ambiente social, nomeadamente à influência dos pares.

As associações reportadas neste estudo podem apontar para diferentes tipos de explicações. A influência observada das dimensões ambientais sugere que pais com percepções positivas do seu ambiente quotidiano, tanto no que respeita aos atributos relativos ao ambiente construído - incluindo-se neste o acesso a destinos úteis e interessantes, a disponibilidade e qualidade de equipamentos e infra-estruturas desportivas e recre- 
ativas, a disponibilidade e manutenção de passeios e ciclovias, etc - como aos do social - considerando o seu lugar de residência como calmo e seguro - provavelmente acreditam que os seus filhos vivem numa área agradável, que thes oferece oportunidades seguras e adequadas, um lugar que propicia o usufruto dos seus espaços públicos, permitindo, pois, que os seus filhos desenvolvam atividades ao ar livre e se movimentem de forma ativa na área (transporte ativo) (TIMPERIo et al., 2005; CARVER et al., 2010). Parece também provável que as próprias crianças, especialmente as mais velhas, sentindo-se seguras, e percepcionando de modo positivo o seu ambiente quotidiano, tenham maior propensão a usufruir dos espaços públicos e abertos, desenvolvendo nestes variadas atividades, com consequências positivas no seu peso.

Porque não são estas associações evidentes para os meninos? A questão remete talvez para as diferentes interacções e usos do espaço feitos por meninas e meninos. Os "espaços de vida" e os "espaços vividos" são certamente diferentes para os dois sexos, que percepcionam e constroem diferentemente os seus espaços. Esta distinta apropriação está patente, por exemplo, nos diferentes comportamentos contextuais desenvolvidos por ambos. Os rapazes, tradicionalmente mais propensos a "jogos de bola" em equipa, talvez sejam menos influenciados pela disponibilidade de infra-estruturas e equipamentos desportivos existentes na área (por exemplo, ginásios), pela existência de "coisas interessantes para ver", pela manutenção dos passeios ou até pela estética, enquanto as meninas, preferindo atividades como dança, ballet, ginástica e, possivelmente, caminhadas, serão mais influenciáveis pela provisão de equipamentos onde possam praticar essas atividades, pela estética geral da área e pelo estado dos passeios. Outra possibilidade é a de haver atitudes parentais diferenciadas consoante o sexo dos filhos. É possível que os pais sejam mais permissivos com os seus filhos rapazes, concedendo-lhes mais liberdade e permitindo-lhes um maior usufruto do espaço público, por os considerarem menos vulneráveis do que as crianças do sexo feminino. Esse comportamento diferenciado pode suceder mesmo em áreas consideradas pouco convenientes, que podem assim ficar "interditas" às meninas, mas acessíveis aos meninos. Todavia, estas questões encontram-se no campo das hipóteses, e terão que ser retomadas e desenvolvidas em estudos posteriores.

O que é inquestionável do estudo até agora efetuado é que a oferta de modalidades desportivas, quer ao nível da diversidade, quer ao nível da quantidade disponível, penaliza fortemente as crianças do sexo feminino, que vêem assim constrangidas as oportunidades de praticar exercício físico. Até que ponto é que essa menor oportunidade pode justificar os menores níveis de atividade física consistentemente observados para as crianças do sexo feminino por diferentes autores (Sallis et al., 2000; SAntos et al., 2009) e ser um risco adicional para os maiores níveis de excesso de peso e obesidade verificado nas meninas? A questão não se encontra ainda abordada, merecendo uma investigação aprofundada, à semelhança de algumas já efetuadas tendo em conta o estatuto socioeconómico. Refira-se, como exemplo, o estudo desenvolvido por GORDON-LARSEn et al. (2006), onde os autores, estudando o acesso de diferentes grupos socioeconómicos às oportunidades de prática de exercício físico, concluíram pelo pior acesso dos grupos mais desfavorecidos.

Quadro III

Oferta (tipo e número) de modalidades destinadas à prática de desporto formal infantil em Coimbra (2009)

\begin{tabular}{|c|c|c|c|c|c|}
\hline \multicolumn{6}{|c|}{ Género a que se destina } \\
\hline \multicolumn{2}{|c|}{ Mistas } & \multicolumn{2}{|c|}{ Tendencialmente masculinas } & \multicolumn{2}{|c|}{ Tendencialmente femininas } \\
\hline Tipo (10) & Número & Tipo (8) & Número & Tipo (3) & Número \\
\hline Remo & 1 & Krav-maga & $1^{\mathrm{a}}$ & Dança & 1 \\
\hline Ténis & 1 & Pólo aquático & $1^{\mathrm{a}}$ & Ballet & 5 \\
\hline Golfe & 1 & Canoagem & $1^{\mathrm{a}}$ & Ginástica & 6 \\
\hline Jiu-jitsu & 1 & Hóquei & 2 & & \\
\hline Andebol & 1 & Rugby & 2 & & \\
\hline Equitação & 2 & Basquetebol & $2^{b}$ & & \\
\hline Capoeira & 2 & Futebol & 5 & & \\
\hline Judo & 4 & Futsal & $9^{b}$ & & \\
\hline Karate & 7 & & & & \\
\hline Natação & 12 & & & & \\
\hline Total & 32 & & 23 & & 12 \\
\hline
\end{tabular}

a Frequentadas exclusivamente por meninos

${ }^{\mathrm{b}}$ Disponibilidade de uma equipa feminina 
Neste caso concreto, sugere-se desde já a existência de uma segregação sexual no acesso às oportunidades de prática desportiva e uma profunda desadequação entre as necessidades das crianças do sexo feminino e as oportunidades oferecidas pelos seus espaços de vida.

\section{Conclusão}

Este estudo encontrou um importante conjunto de resultados que possibilitam e suportam um não menos importante conjunto de conclusões. Em primeiro lugar, a evidência de que é possível atenuar ou até reverter a tendência generalizada para o aumento de peso que se tem verificado através de intervenções ambientais. Melhorar a saúde das atuais crianças e dos futuros adultos não é apenas uma questão da esfera da saúde e das políticas de saúde; é também uma questão de planeamento urbano. Melhorar a qualidade do ambiente construído e social, aumentando a disponibilidade de equipamentos recreativos e desportivos, a disponibilidade e o estado de manutenção dos passeios e das ciclovias, promovendo a diversificação nos usos do solo, iluminando e cuidando dos espaços públicos, diminuindo a intensidade do tráfego rodoviário, bem como a sua velocidade, aumentando assim a segurança real e percepcionada, são medidas concretas que podem promover uma maior interacção das crianças com os seus espaços de vida. Caminhar, andar de bicicleta, brincar em espaços públicos, sejam praças, parques ou jardins, só é possível se o espaço for agradável e transmitir segurança.

Numa análise mais fina, emerge um segundo e incisivo conjunto de conclusões. Verificando-se que as meninas têm maior prevalência de excesso de peso e obesidade, e que o seu peso é mais sensível à influência do ambiente, as medidas referidas anteriormente ganham uma relevância acrescida. Porém, verifica-se uma situação paradoxal, uma vez que estas crianças usufruem de menos oportunidades para praticar exercício físico face aos seus congéneres do sexo masculino. A análise efetuada à oferta de modalidades desportivas revelou a existência de uma situação insidiosa e injusta de discriminação ambiental das meninas, que pode estar a contribuir para as diferenças conhecidas nos padrões de excesso de peso/obesidade e atividade física por género. Esta situação de injustiça pode e deve ser combatida, adequando o ambiente às necessidades específicas da sua população. Considerar as diferenças existentes entre grupos e indivíduos e dis- criminar positivamente aqueles que pela sua condição socioeconómica, sexual, ou outra, são mais vulneráveis e desfavorecidos, parece fundamental para uma sociedade mais saudável. Saúde, justiça e equidade não são dissociáveis.

\section{Bibliografia}

Burdette L. e Whitaker, C. (2003) - "Neighborhood playgrounds, fast food restaurants, and crime: relationships to overweight in low-income preschool children". Preventive Medicine, 38, pp. 57-63.

Carver, A.; Timperio, A.; Hesketh, K. e Crawford, D., (2010) - "Are children and adolescents less active if parents restrict their physical activity and active transport due to perceived risk?" Social Science and Medicine 70, pp. 1799-1805.

Cerin, E.; Leslie, E.; Owen, N. e Bauman, A. (2007) - "Applying GiS in physical activity research: community walkability and walking behaviours". In: LAI, P. e MAK A. (Eds.) GIS for Health and the Environment, Springer, Berlin, Heidelberg, New York, pp. 72-89.

Cohen, D.; Finch, B.; Bower, A. e SAStry, N. (2006) - "Collective efficacy and obesity: The potential influence of social factors on health." Social Science and Medicine, 62(3), pp. 769-778.

Cole, T.; Bellizzı, M.; Flegal, K. e Dietz, W. (2000) - "Establishing a standard definition for child overweight and obesity worldwide: international survey. BMJ 320, 1240-doi: 10.1136/bmj.320.7244.1240.

Cummins, S.; Macintyre, S.; Davidson, S. e Ellaway, A. (2005) "Measuring neighbourhood social and material context: generation and interpretation of ecological data from routine and non-routine sources." Health \& Place 11 (3) pp. 249-260.

Evenson, K.; Birnbaum, A.; Bedimo-Rung, A.; Sallis, J.; Voorhess, C. e Elder, J. (2006) - "Girls' perception of physical environmental factors and transportation: reliability and association with physical activity and active transport to school. International Journal of Behavioral Nutrition and Physical Activity 3 (28), doi: 10.1186/1479-5868-3-28.

Finucane, M.; Stevens, G.; Cowan, M.; Danaei, F.; Lin, J.; Paciorek, C.; Singh, G.; Gutierrez, H.; Lu, Y.; Bahalim, A.; Farzadfar, F.; RIley, L. e Ezzatı, M. (2011) - "National, regional, and global trends in body-mass index since 1980: systematic analysis of health examination surveys and epidemiological studies with 960 country-years and 9.1 million participants." Lancet 377, pp. 557-567.

Frank, L.; Andresen, M. e Schmid, T. (2004) - "Obesity relationships with community design, physical activity, and time 
spend in cars. American Journal of Preventive Medicine, 27 (2), pp. 87-96.

Frank, L.; Kerr, J.; Chapman, J. e Sallis, J.F. (2007) - "Urban form relationships with walk trip frequency and distance among youth". American Journal of Health Promotion 21 (4), pp. 305-311.

Giles-Corti, B. e Donovan, R. J. (2003) - "Relative influences of individual, social environmental, and physical environmental correlates of walking." American Journal of Public Health 93, pp. 1583-1589.

Gordon-Larsen, P.; Nelson, M. C.; Page, P. e Popkin, B. M. (2006) "Inequality in the built environment underlies key health disparities in physical activity and obesity." Pediatrics 117, pp. 417-424.

Hume, C.; Salmon, J. e Ball, K. (2005) - "Children's perceptions of their home and neighborhood environments, and their association with objectively measured physical activity: a qualitative and quantitative study." Health Educ Res 20 (1), pp. 1-13.

IPS (2002) - Physical activity prevalence study environmental survey module. Available online: htpp://www-rohan. sdsu.edu/faculty/sallis/IPAQIPS.pdf.

Irving, J.; He, M.; Sangster Bouck, L.; Tucker, P. e Pollett, G. (2005) - "Preschoolers' physical activity behaviours; parents' perspectives." Canadian Journal of Public Health 96 (4), pp. 299-303.

Kim, D.; Subramanian, S.; Gortmaker, S. e Kawachi, I. (2006) - "US state-and county level social capital in relation to obesity and physical inactivity: multilevel, multivariable analysis." Social Science and Medicine, 63, pp. 10451059.

Leslie, E.; Coffee, N.; Frank, L.; Owen, N; Bauman, A. e Hugo, G. (2007) - "Walkability of local communities: using geographical information systems to objectively assess relevant environmental attributes." Health \& Place, 13, pp. 111-122.

MACINTYRE, S. (2007) - "Deprivation amplification revisited, or, is it always true that poorer places have poorer access to resources for healthy diets and physical activity?" International Journal of Behavioral Nutrition and Physical Activity, 4(32). doi:10.1186/1479-5868-43232.

McGinn, A.; Evenson, K.; Herring, A. e Huston, S. (2007) - "The relationship between leisure, walking, and transportation activity with the natural environment. "Health \& Place 13, pp. 588-602.

Mladovsky, P.; Allin, S.; Masseria, C.; Hernández-Quevedo, C.; McDaid, D. e Mossialos, E. (2009) - "Health in the European Union. Trends and analysis." Copenhagen: European Observatory on Health Systems and Policies, WHO Regional Office for Europe.
Mota, J.; Almeida, M.; Santos, P. e Ribeiro, J.C. (2005) - "Perceived neighborhood environments and physical activity in adolescents." Preventive Medicine 41, pp. 834-836.

NogueIRA, H. (2010) - "Deprivation amplification and health promoting resources in the context of a poor country." Social Science and Medicine, 70, pp. 1391-1395.

Nogueira, H. (2009) - "Healthy communities: the challenge of social capital in the Lisbon Metropolitan Area. "Health \& Place 15, pp. 133-139.

Padez, C.; Fernandes, T.; Mourão, I.; Moreira, P. e Rosado, V. (2004) "Prevalence of overweight and obesity in 7-9-year-old Portuguese children: Trends in body mass index from 1970-2002". American Journal of Human Biology 16: 6, pp. 670-678.

Poortinga, W. (2006) - "Perceptions of the environment, physical activity, and obesity." Social Science and Medicine 63, pp. 2835-2846.

Rundle, A.; Field, S.; Park, Y.; Freeman, L.; Weiss, C. e Neckerman, K. (2009) - "Personal and neighborhood socioeconomic status and indices of neighborhood walk-ability predict body mass index in New York City." Social Science and Medicine 63, pp. 2835-2846.

Sallis, J.; Prochaska, J. e Taylor, W. (2000) - "A review of correlates of physical activity of children and adolescents." Med Sci Sports Exerc 32, pp. 963-975.

Santos, M.; Vale, M.; Miranda, L. e Mota, J. (2009) - "Sociodemographic and perceived environmental correlates of walking in Portuguese adults - A multilevel analysis." Health \& Place 15, pp. 1094-1099.

Santos, R. (1999) - "Cronbach's Alpha: A Tool for Assessing the Reliability of Scales." Available online: http://www.joe. org/joe/1999april/tt3.html). Texas, Texas University.

Santos, R.; Silva, P.; Santos, P.; Ribeiro, J. e Mota, J. (2008) - "Physical activity and perceived environmental attributes in a sample of Portuguese adults: results from the Azorean Physical Activity and Health Study." Preventive Medicine 47, pp. 83-88.

Timperio A.; Crawford, D.; Telford, A. e Salmon, J. (2004) - "Perceptions of the local neighbourhood and walking and cycling among children." Preventive Medicine 38, pp. 39-47.

Timperio, A.; Salmon, J.; Telford, A. e Crawford, D. (2005) "Perceptions of local neighbourhood environments and their relationship to childhood overweight and obesity." International Journal of Obesity 29, pp. 170-175.

Tucker, P.; Irwin, J.; Gilliland, J.; He, M.; Larsen, K. e Hess, P. (2009) - "Environmental influences on physical activity levels in youth." Health \& Place 15, pp. 357-363.

WANG, G. e Dietz, WH. (2002) - "Economic burden of obesity in youths aged 6 to 17 years: 1979-1999." Pediatrics 109, pp. E81-E86.

WANG, Y. e Lobstein, T. (2006) - "Worldwide trends in childhood obesity." Int J Pediatr Obesity 1, pp. 11-25. 
World Health Organization (2000) - Obesity. Prevention and managing the global epidemic: report of a WHO consultation on obesity. WHO Technical Report Series, n 894, Geneve, WHO. 